\title{
Selection of co-transformed Dendrobium Sonia 17 using hygromycin and green fluorescent protein
}

\begin{abstract}
Dendrobium Sonia 17 calluses were used for co-transformation study using particle bombardment. The bombarded transformed callus tissues were selected using half-strength MS medium containing $25 \mathrm{mg} \mathrm{dm}-3$ hygromycin. Expression of green fluorescent protein (GFP) was observed in the callus and protocorm-like body (PLBs) tissues survived on the selection medium. The presence of green fluorescence protein (sgfp), hygromycin-Bphosphotransferase (hptII) and $\beta$-glucuronidase (uidA) genes in the transformed tissues were verified using PCR, Southern blot and dot blot analyses. Based on the results from PCR and expression of sgfp and uidA genes in the calluses and PLBs survived from hygromycin selection, we reported the co-transformation of sgfp, hptII and uidA genes into Dendrobium Sonia 17. GFP-expressing tissues were also observed in the regenerated transformed plantlets.
\end{abstract}

Keyword: Bombardment; $\beta$-glucuronidase; PCR; Phosphotransferase; Southern blot 